\title{
Sufficient Conditions for Evasion in a Linear Differential Game
}

\author{
Nodir Umrzakov \\ Institute of Mathematics and IT \\ Durman yuli, 29, 100125, Tashkent, Uzbekistan \\ Tel: 998-74-970-7150 E-mail: umrzaqov2010@mail.ru \\ Gafurjan Ibragimov (Corresponding author) \\ Department of Mathematics, Faculty of Science, Universiti Putra Malaysia \\ 43400, Serdang, Selangor, Malaysia \\ Tel: 60-3-8946-6818 E-mail: gafur@science.upm.edu.my
}

\begin{abstract}
Received: August 17, 2011 Accepted: September 22, 2011 Published: November 1, 2011
doi:10.5539/jmr.v3n4p168 URL: http://dx.doi.org/10.5539/jmr.v3n4p168
\end{abstract}

\begin{abstract}
We study a linear evasion differential game in $\mathbb{R}^{2}$. Control sets of players, the pursuer and the evader, are compact subsets of $\mathbb{R}^{2}$. The terminal set of the game is the origin. The game is considered to be completed if the state of the system, $z(t)$, reaches the origin. If $z(t)$ never reaches the origin, then we say that evasion is possible in the game. We obtained weaker conditions for evasion than conditions obtained by other researches. We give some illustrative examples which show the advantage of our conditions.
\end{abstract}

Keywords: Differential game, Evasion, Strategy, Control

\section{Statement of the problem}

Dynamics of the state vector $z(t)$ is described in $\mathbb{R}^{2}$ by the following system of equations

$$
\dot{z}=C z-u+v+a, z(0)=z_{0} \neq 0,
$$

where

$$
C=\left[\begin{array}{ll}
c_{11} & c_{12} \\
c_{21} & c_{22}
\end{array}\right], \quad u=\left[\begin{array}{l}
u_{1} \\
u_{2}
\end{array}\right], v=\left[\begin{array}{l}
v_{1} \\
v_{2}
\end{array}\right], a=\left[\begin{array}{l}
a_{1} \\
a_{2}
\end{array}\right],
$$

$u$ is control parameter of the pursuer, $v$ is that of the evader, $C$ is a constant matrix, $a$ is a constant vector. The control parameters $u$ and $v$ are chosen from the control sets $U$ and $V$, respectively, which are compact subsets of $\mathbb{R}^{2}$.

Definition 1. A measurable function $u(t), u:[0, \infty) \rightarrow U$, is called a control of the pursuer.

Definition 2. A measurable function $v(t), v:[0, \infty) \rightarrow V$, is called a control of the evader.

Definition 3. A function $V_{0}=V_{0}(z, u), V_{0}: \mathbb{R}^{2} \times U \rightarrow V$, such that for any control of the pursuer $u(\cdot)$ the system

$$
\dot{z}=C z-u+V_{0}(z, u)+a, z(0)=z_{0},
$$

has a unique solution $z(t), t \geq 0$, and $V_{0}(z(t), u(t)), t \geq 0$, is measurable, is called a strategy of the evader.

Definition 4. If there exists a strategy of the evader $V_{0}$ such that for any control of the pursuer $u(t), t \geq 0$, the solution of the initial value problem (2), $z(t), t \geq 0$, with $z(0)=z_{0} \neq 0$, will not pass through the origin, then we say that evasion is possible in the game (1).

Problem. Find conditions on the matrix $C$, the control sets $U$ and $V$, and the vector $a$ for which evasion is possible in the game (1).

\section{Related works}

Evasion is an important branch of the Differential Game Theory. Many researches are devoted to evasion problems (see, for example, [1-8]). The evasion problem first formulated by (Pontryagin, 1969), (Pontryagin, 1971) and he solved it 
under "rotatability" and "advantage" conditions. (Pontryagin, 1971) formulated the following problem called $\mu$-problem: is it possible to prove an evasion theorem in case of $\mu=1$, where $\mu$ represents advantage of the evader. However, a positive solution of the problem has still not been obtained. It should be noted that in some particular cases the problem was solved by (Kramarovskii, 1995), (Kuchkarov, 2002), (Satimov, 2000).

(Satimov, 2000) and (Kuchkarov, 2002) proved that evasion is possible in the game (1) if the following two conditions hold:

1. there doesn't exist a straight line $L$ passing through the origin, which satisfies the inclusion $e^{t C} V \subset L$ for all sufficiently small positive $t$ (the rotatability condition),

2. the inclusion $U \subset V$ holds (the advantage condition).

(Kramarovskii, 1995), and (Satimov, 2000) showed that if the advantage condition and the inequality

$$
\min _{u \in U} \max _{v \in V}\left|\left(u_{1}-v_{1}\right) a_{2}-\left(u_{2}-v_{2}\right) a_{1}\right|>0
$$

hold, then evasion is possible in the game (1).

In this paper we'll give some conditions weaker than that in the above for which the evasion is possible. Also we'll give some examples for which game is described by a system of the form (1), and both the rotatability and the advantage conditions are not satisfied, but our conditions are satisfied.

\section{Main Result}

Since a parallel translation of any of the sets $U$ and $V$ in the space $\mathbb{R}^{2}$ can be compensated by a change in the vector $a$, we can assume that the set $V$ contains the origin.

Theorem. Let the advantage condition and the following conditions hold:

C1. The system (1) cannot be reduced to the form

$$
\left\{\begin{array}{l}
\dot{z}_{1}=\bar{c}_{11} z_{1} \\
\dot{z}_{2}=\bar{c}_{21} z_{1}+\bar{c}_{22} z_{2}-\bar{u}+\bar{v}+\bar{a}
\end{array}\right.
$$

by rotating the coordinate system, where $\bar{c}_{11}, \bar{c}_{21}, \bar{c}_{22}, \bar{a}$ are some constants, and $\bar{u} \in \bar{U} \subset \mathbb{R}^{1}, \bar{v} \in \bar{V} \subset \mathbb{R}^{1}$.

C2. The set $V$ contains at least two points.

Then evasion is possible in the game (1).

Proof. We analyze the following cases:

Case 1. There is a straight line $L$ which passes through the origin and contains the set $V$.

Case 2. There is no straight line containing the set $V$ and passing through the origin.

Study the case 1. We rotate the coordinate system so that $z_{2}$-axis coincides with the straight line $L$. We denote new coordinate axes again by $z_{1}, z_{2}$. Clearly, in this coordinate system, equation (1) takes the form

$$
\left\{\begin{array}{l}
\dot{z}_{1}=c_{11} z_{1}+c_{12} z_{2}+a_{1} \\
\dot{z}_{2}=c_{21} z_{1}+c_{22} z_{2}-\bar{u}+\bar{v}+a_{2}
\end{array}\right.
$$

where $\bar{u} \in \bar{U} \subset \mathbb{R}^{1}, \bar{v} \in \bar{V} \subset \mathbb{R}^{1}$. Letting $\bar{v}=\bar{u}$ in (4) we obtain

$$
\left\{\begin{array}{l}
\dot{z}_{1}=c_{11} z_{1}+c_{12} z_{2}+a_{1} \\
\dot{z}_{2}=c_{21} z_{1}+c_{22} z_{2}+a_{2}
\end{array}\right.
$$

There is only one trajectory, denoted $z_{1}=\phi_{1}(t), z_{2}=\phi_{2}(t)$, passing through the origin. This trajectory is defined for all $t \in(-\infty,+\infty)$. We assume that $\phi_{1}(0)=\phi_{2}(0)=0$.

Let $\Pi=\left\{\left(\phi_{1}(t), \phi_{2}(t)\right): t \leq 0\right\}$. It is clear that if $z_{0} \notin \Pi$, then letting $\bar{v}=\bar{u}$ and using the fact that trajectories of (5) do not intersect we get $z(t) \neq 0, t \geq 0$, meaning that evasion is possible in the game (4).

Note that if $a_{1}=a_{2}=0$, then $\phi_{1}(t) \equiv 0, \phi_{2}(t) \equiv 0$, and $\Pi$ consists of the only point $(0,0)$, therefore, from the point $z_{0} \neq 0$ evasion is possible in the game (4) and hence in the game (1) too. 
In the sequel, we assume that $a \neq 0$ and $z_{0} \in \Pi \backslash\{0\}$. Then either $a_{1} \neq 0$ or $a_{1}=0, a_{2} \neq 0$. Let $a_{1} \neq 0$. For definiteness we assume that $a_{1}>0$. The case $a_{1}<0$ can be examined in a similar way. As $\dot{\phi}_{1}(0)=a_{1}>0$, then by the Implicit Function Theorem the equation $z_{1}=\phi_{1}(t)$ can be solved for $t$ at some neighborhood of the point 0 , i.e., $t=f\left(z_{1}\right),\left|z_{1}\right| \leq \delta, \delta>0$. Then we obtain continuously differentiable function

$$
z_{2}=\phi_{2}(t)=\phi_{2}\left(f\left(z_{1}\right)\right)=h\left(z_{1}\right),\left|z_{1}\right| \leq \delta .
$$

Clearly, the equation

$$
z_{2}=h\left(z_{1}\right),-\delta \leq z_{1} \leq 0
$$

expresses a part of the line $\Pi$, denoted $l$, which connects $\Pi$ with the origin.

$1^{\circ}$. We show that if $z_{0} \in \Pi \backslash l$, then evasion is possible in the game (4). The strategy of the evader consists of three parts. The first part comprises the formula

$$
\bar{v}(t)=\bar{u}(t) \text { if } z_{1}(t) \neq-\frac{\delta}{2},
$$

where $z_{1}(t)$ is the first coordinate of the solution $z(t)=\left(z_{1}(t), z_{2}(t)\right)$ of (4) with the initial condition $z(0)=z_{0}$. Here $z(t)$ moves along $\Pi$ towards 0 . Hence $z_{1}\left(t_{0}\right)=-\frac{\delta}{2}$ at some time $t_{0}>0$. Without loss of generality we now consider the point $\left(-\frac{\delta}{2}, h\left(-\frac{\delta}{2}\right)\right)$ as the initial position of $z(t)$ at $t_{0}=0$ and we set $z_{0}=\left(-\frac{\delta}{2}, h\left(-\frac{\delta}{2}\right)\right)$.

Since the solution of the system (4) is continuous, therefore there exists a number $\varepsilon>0$ such that for any admissible controls of players $\bar{u}(t)$ and $\bar{v}(t)$ the solution of the system $(4), \bar{z}(t)=\left(\bar{z}_{1}(t), \bar{z}_{2}(t)\right), 0 \leq t \leq \varepsilon$, at $\bar{u}=\bar{u}(t), \bar{v}=\bar{v}(t)$ with the initial position $z_{0}$ at $t_{0}=0$ cannot reach neither the point 0 nor the point $(-\delta, h(-\delta))$ on $[0, \varepsilon]$.

We now turn to the construction of the second part of the evader's strategy. We let

$$
\bar{v}(t) \neq \bar{u}(t), v(t) \in V, t \in[0, \varepsilon] .
$$

According to the condition $\mathrm{C} 2$ of the theorem such admissible control $\bar{v}(t)$ exists. We show that $\bar{z}(t) \notin \Pi$ at some $t=t_{1} \in(0, \varepsilon]$. Assume the contrary. Let $\bar{z}(t) \in \Pi$ for all $t \in[0, \varepsilon]$. Then by construction of the line $l$ we have $\bar{z}(t) \in l$ for all $t \in[0, \varepsilon]$. Therefore by (6) we obtain

$$
\bar{z}_{2}(t)=h\left(\bar{z}_{1}(t)\right), \dot{\bar{z}}_{2}(t)=h^{\prime}\left(\bar{z}_{1}(t)\right) \cdot \dot{\bar{z}}_{1}(t) .
$$

Then by (4) we have

$$
c_{21} \bar{z}_{1}+c_{22} \bar{z}_{2}-\bar{u}(t)+\bar{v}(t)+a_{2}=h^{\prime}\left(\bar{z}_{1}(t)\right)\left[c_{11} \bar{z}_{1}+c_{12} \bar{z}_{2}+a_{1}\right] .
$$

Since

$$
\bar{z}(t) \in l=\left\{\left(\phi_{1}(s), \phi_{2}(s)\right) \mid s \in[-\delta, 0]\right\},
$$

$\bar{z}(t)=\left(\phi_{1}(s), \phi_{2}(s)\right)$ for some $s \in[-\delta, 0]$. Substitute it into (7) to obtain

$$
c_{21} \phi_{1}(s)+c_{22} \phi_{2}(s)-\bar{u}(t)+\bar{v}(t)+a_{2}=h^{\prime}\left(\phi_{1}(s)\right)\left[c_{11} \phi_{1}(s)+c_{12} \phi_{2}(s)+a_{1}\right] .
$$

By (5)

$$
\dot{\phi}_{1}(s)=c_{11} \phi_{1}(s)+c_{12} \phi_{2}(s)+a_{1}, \dot{\phi}_{2}(s)=c_{21} \phi_{1}(s)+c_{22} \phi_{2}(s)+a_{2} .
$$

Therefore by (8)

$$
\dot{\phi}_{2}(s)-\bar{u}(t)+\bar{v}(t)=h^{\prime}\left(\phi_{1}(s)\right) \dot{\phi}_{1}(s) .
$$

On the other hand, we have the identity $\phi_{2}(t)=h\left(\phi_{1}(t)\right), t \in[-\delta, 0]$, consequently, $\dot{\phi}_{2}(t)=h^{\prime}\left(\phi_{1}(t)\right) \dot{\phi}_{1}(t)$. If here we let $t=s$, then from (9) we obtain $-\bar{u}(t)+\bar{v}(t)=0$. However, by construction $\bar{v}(t) \neq \bar{u}(t), 0 \leq t \leq \varepsilon$. Contradiction.

Thus, if $a_{1}>0$ and $z_{0} \in \Pi \backslash l$, then we obtain $\bar{z}\left(t_{1}\right) \notin \Pi$ at some $t_{1} \in(0, \varepsilon]$. Then, clearly, letting

$$
\bar{v}(t)=\bar{u}(t), t \geq t_{1},
$$

which is the third part of the evader's strategy, we get $\bar{z}(t) \neq 0, t \geq t_{1}$, that is, evasion is possible from the initial position $z_{0}$ in the game (4).

$2^{\circ}$. We show that evasion is possible in the case $z_{0} \in l \backslash\{0\}$. Indeed, by continuity of the trajectory of the system (4) there exists $\varepsilon>0$ such that for any admissible controls $\bar{u}(t)$ and $\bar{v}(t)$ the trajectory $z(t)$ of the system (4) at $\bar{u}=\bar{u}(t), \bar{v}=\bar{v}(t)$ with 
$z(0)=z_{0} \in l \backslash\{0\}$ can reach neither the point $(-\delta, h(-\delta))$ nor the point 0 . From here arguments are similar to those in the case $z_{0} \in \Pi \backslash l$.

We now study the case where $a_{1}=0, a_{2} \neq 0$. From (5) we obtain that $\dot{\phi}_{1}(0)=0, \ddot{\phi}_{1}(0)=c_{12} a_{2}$. Here $c_{12} \neq 0$, otherwise the system (4) takes the form contradicting to the condition C1. Hence, $c_{12} a_{2} \neq 0$. For definiteness we assume that $c_{12} a_{2}<0$ (the case $c_{12} a_{2}>0$ is considered in a similar way). Then relations $\dot{\phi}_{1}(0)=0, \ddot{\phi}_{1}(0)<0$ imply that the function $z_{1}=\phi_{1}(t)$ attains its local minimum at $t=0$ and increases monotonously on some interval $[-\tau, 0], \tau>0$. Therefore the equation $z_{1}=\phi_{1}(t)$ can be solved for $t: t=f\left(z_{1}\right), z_{1} \in[-\varepsilon, 0]$. Substituting this into the equation $z_{1}=\phi_{2}(t)$ we get the function $z_{2}=\phi_{2}\left(f\left(z_{1}\right)\right)=h\left(z_{1}\right)$ which represents a part of $\Pi$, which connects $\Pi$ with the origin. Further arguments are similar to those in the case $a_{1} \neq 0$.

Thus, we can conclude that if the set $V$ is contained in a straight line $L$ passing through the origin then evasion is possible.

We analyze now the case 2: there is no straight line, which passes through the origin and contains the set $V$. Then $V$ contains two linearly independent vectors

$$
v^{\prime}=\left(v_{1}^{\prime}, v_{2}^{\prime}\right), \quad v^{\prime \prime}=\left(v_{1}^{\prime \prime}, v_{2}^{\prime \prime}\right)
$$

If $a=0$ then we set $v(t)=u(t)$. Then the system (1) reduces to $\dot{z}=C z, z(0)=z_{0} \neq 0$, and, clearly, $z(t) \neq 0, t \geq 0$. This means the evasion is possible in the game (1).

From now on we assume that $a=\left(a_{1}, a_{2}\right) \neq 0$. Let

$$
N(u, v)=\left|\begin{array}{ll}
v_{1}-u_{1} & a_{1} \\
v_{2}-u_{2} & a_{2}
\end{array}\right| .
$$

We show that

$$
N=\min _{u \in U} \max _{v \in V}|N(u, v)|>0 .
$$

Assume the contrary. Let $N=0$. Then

$$
\left|\begin{array}{ll}
v_{1}-u_{10} & a_{1} \\
v_{2}-u_{20} & a_{2}
\end{array}\right|=0
$$

for all $v=\left(v_{1}, v_{2}\right) \in V$ and some $u_{0}=\left(u_{10}, u_{20}\right) \in U$. This equality shows that vectors $v-u_{0}$ and $a \neq 0$ are parallel for all $v \in V$. In particular, the vectors $v^{\prime}-u_{0}$ and $v^{\prime \prime}-u_{0}$ are parallel to $a$. This contradicts to the condition that vectors (10) are linearly independent. Therefore (11) holds.

Let $v_{0}(u)$ is the least lexicographic solution of the equation

$$
|N(u, v)|=\max _{v \in V}|N(u, v)| .
$$

If $u(t), 0 \leq t \leq 1$ is a measurable function then so is $v_{0}(u(t)), 0 \leq t \leq 1$ (Mishchenko, 1973). By (11)

$$
\left|N\left(u(t), v_{0}(t)\right)\right| \geq N
$$

As $a \neq 0$, then either $a_{1} \neq 0$ or $a_{1}=0, a_{2} \neq 0$. In the former case, a part of $\Pi$ connecting it with 0 can be represented in the form (6). We show that $z(t) \notin \Pi$ at some $t_{1} \in[0, \varepsilon]$. If we assume the contrary, then analysis similar to that in the case 1 with $a_{1} \neq 0$ shows that

$$
v_{20}(u(t))-u_{2}(t)=h^{\prime}\left(z_{1}(t)\right)\left[v_{10}(u(t))-u_{1}(t)\right]
$$

where $\left(u_{1}(t), u_{2}(t)\right)=u(t), 0 \leq t \leq \varepsilon$, is an admissible control chosen by the pursuer, $\left(v_{10}(u(t)), v_{20}(u(t))\right)=v_{0}(u(t))$, $0 \leq t \leq \varepsilon$, is the function defined by $(12),\left(z_{1}(t), z_{2}(t)\right)=z(t), 0 \leq t \leq \varepsilon$, is the trajectory of the system (1) at $u=u(t)$, $v=v_{0}(t)$, with either $z(0)=z_{0} \in \Pi \backslash l$ or $z_{0} \in l \backslash\{0\}$.

By the definition of $h\left(z_{1}\right)$ we obtain that $h^{\prime}\left(\phi_{1}(t)\right)=\frac{\dot{\phi}_{2}(t)}{\dot{\phi}_{1}(t)}$ and hence $h^{\prime}(0)=\frac{a_{2}}{a_{1}}$. From this and continuity of $h^{\prime}\left(z_{1}\right)$ there exists $\delta_{0}>0$ such that for all $z_{1},-\delta_{0} \leq z_{1} \leq 0$,

$$
\left|h^{\prime}\left(z_{1}\right)-\frac{a_{2}}{a_{1}}\right|<\frac{N}{2\left|a_{1}\right| M},
$$


where $M=\max _{u \in U, v \in V}\left|v_{1}-u_{1}\right|$. Based on (13), (14) and (15) we estimate $\left|N\left(u(t), v_{0}(t)\right)\right|$ :

$$
\begin{aligned}
N \leq\left|N\left(u(t), v_{0}(t)\right)\right| & =\left|a_{2}\left(v_{10}(t)-u_{1}(t)\right)-a_{1}\left(v_{20}(t)-u_{2}(t)\right)\right| \\
& =\left|a_{1}\right| \cdot\left|\frac{a_{2}}{a_{1}}\left(v_{10}(t)-u_{1}(t)\right)-\left(v_{20}(t)-u_{2}(t)\right)\right| \\
& =\left|a_{1}\right| \cdot\left|\frac{a_{2}}{a_{1}}\left(v_{10}(t)-u_{1}(t)\right)-h^{\prime}\left(z_{1}(t)\right)\left(v_{10}(t)-u_{1}(t)\right)\right| \\
& =\left|a_{1}\right| \cdot\left|\frac{a_{2}}{a_{1}}-h^{\prime}\left(z_{1}(t)\right)\right| \cdot\left|v_{10}(t)-u_{1}(t)\right| \leq\left|a_{1}\right| \cdot \frac{N}{2\left|a_{1}\right| M} \cdot M=\frac{N}{2},
\end{aligned}
$$

which is impossible. Therefore $z\left(t_{1}\right) \notin \Pi$ at some $t_{1} \in[0, \varepsilon]$. We can now proceed by letting

$$
v(t)=u(t), t \geq t_{1} .
$$

Then clearly, $z(t) \neq 0, t \geq 0$.

Similar arguments apply to the case $a_{1}=0, a_{2} \neq 0$. The equation of the line $l$ now has the form

$$
z_{1}=g\left(z_{2}\right),-\delta \leq z_{2} \leq 0, \delta>0 .
$$

We can now proceed analogously to the case $a_{1} \neq 0$. We obtain the equation

$$
v_{10}(t)-u_{1}(t)=h^{\prime}\left(z_{2}(t)\right)\left[v_{20}(t)-u_{2}(t)\right],
$$

where $\left(u_{1}(t), u_{2}(t)\right)=u(t), 0 \leq t \leq \varepsilon$, is an admissible control chosen by the pursuer, $\left(v_{10}(t), v_{20}(t)\right)=v_{0}(t), 0 \leq t \leq \varepsilon$, is the function obtained from (12), $\left(z_{1}(t), z_{2}(t)\right)=z(t), 0 \leq t \leq \varepsilon$, is the trajectory of the system (1) at $u=u(t), v=v_{0}(t)$, with either $z(0)=z_{0} \in \Pi \backslash l$ or $z_{0} \in l\{0\}$. The rest of the proof runs as before. The proof of the theorem is complete.

\section{Comparison of the Conditions}

In this section, we show that the conditions $\mathrm{C} 1$ and $\mathrm{C} 2$ of the theorem are weaker than that in section 2 .

1. Let the rotatablility and advantage conditions (Kuchkarov, 2002), (Satimov, 2000) hold. We show that the conditions of the theorem are satisfied. Indeed, from the condition $0 \in V$ we obtain that the set $V$ contains at least two different points, since otherwise $e^{t C} V=(0,0)$, which contradicts the rotatability condition. We now turn to the condition $\mathrm{C} 1$ of the theorem. The system (1) cannot be reduced to the form given in the condition $\mathrm{C} 1$, for otherwise the fundamental matrix of (1) has the form

$$
e^{t C}=\left(\begin{array}{cc}
e_{11}(t) & 0 \\
e_{21}(t) & e_{22}(t)
\end{array}\right)
$$

and hence $e^{t C} \bar{V}=\left(0, e_{11}(t) \bar{v}\right)$. This means $z_{2}$-axis contains the set $e^{t C} \bar{V}$, which contradicts the rotatability condition.

2. We next show that conditions of works (Kramarovskii, 1995) and (Satimov, 1984), i.e., the inequality (3) and the advantage condition, imply the conditions $\mathrm{C} 1$ and $\mathrm{C} 2$ of the theorem. It follows from the condition $0 \in V$ that $V$ contains at least two different points, since otherwise $U=V=\{(0,0)\}$ and hence the inequality (3) is not satisfied. If we now assume that the system (1) can be reduced to the form given in the condition $\mathrm{C} 1$ by rotating the coordinate system then we obtain that $u_{1}-v_{1}=0, a_{1}=0$. Hence the inequality (3) is not satisfied. Contradiction.

We now give examples that show the advantage of our conditions.

Example 1. Let a differential game is described by the system of equations

$$
\left\{\begin{array}{l}
\dot{z}_{1}=1 \\
\dot{z}_{2}=z_{1}-u+v
\end{array}\right.
$$

where $u, v \in\{0,3\}$ showing that $U$ and $V$ are not convex. We see at once that the conditions of our theorem are satisfied, but those of the works [2-7] are not satisfied.

Example 2. We now consider a differential game described by the system

$$
\left\{\begin{array}{l}
\dot{z}_{1}=z_{2} \\
\dot{z}_{2}=-u+v+1
\end{array}\right.
$$


where $u, v \in\{0,3\}$. A trivial verification shows that the conditions $\mathrm{C} 1$ and $\mathrm{C} 2$ of the theorem are satisfied, but those of the papers (Kramarovskii, 1995) and (Satimov, 1984) are not satisfied.

\section{Acknowledgment}

This research was partially supported by the Research Grant (RUGS) of the Universiti Putra Malaysia, No. 05-04-101005RU.

\section{References}

Kramarovskii V. B. (1995). On some critical cases in quasilinear differential games of evasion. Uzbek Mathematical Journal, 3, 73-79.

Kuchkarov A. Sh., Rikhsiev B. B. (2002). The possibility of avoiding an encounter in a linear differential game of evasion. J. Appl. Math. Mechs. Vol 66, No 2, 233-237. http://dx.doi.org/10.1016/S0021-8928(02)00028-X

Mishchenko Ye. F., Satimov N. Yu. (1973). The problem of avoiding in differential games with non linear controls. Differential Equations, Vol.9. No.10, 1792-1797.

Nikol'skii M. S. (1974). A linear problem of evasion. Dokl. Akad. Nauk SSSR, Vol.218, No 5, 1024-1027.

Pontryagin L. S., and Mishchenko Ye. F. (1969). The problem of avoiding a controlled object by another object. Dokl. Akad. Nauk SSSR, Vol.189, No 4, 721-723.

Pontryagin L. S. (1971). A linear differential game of evasion. R Mat. Inst. Akad. Nauk SSSR, Vol.112, No.1, 30-63.

Satimov N. Yu., Rikhsiev B. B. (2000). Methods for Solving the Evasion Problem in Mathematical Control Theory. Tashkent, Fan.

Satimov N. Yu. (1984). On the theory two dimensional quasilinear differential games of evasion. Controlled systems, 24, 79-84. 